\title{
Fabrication aspects of microwave devices, including ramp-type high-Tc Josephson junctions and log-periodic antennas.
}

\author{
D. Terpstra, A.J.H.M. Rijnders, F.J.G. Roesthuis, D.H.A. Blank, G.J. Gerritsma and H. Rogalla \\ University of Twente, Applied Physics Department, P.O.Box 217, 7500 AE Enschede, The Netherlands.
}

\begin{abstract}
We describe the development of high- $\mathrm{T}_{\mathrm{c}}$ Josephson junction devices for applications at millimeter wave frequencies. These devices consist of ramp type YBCO/PBCO/YBCO Josephson junctions that are equipped with a noble metal log-periodic antenna. Growth conditions of all layers, as well as etching, cleaning and annealing procedures are being optimized, to guarantee well-defined device properties. Lowering the deposition temperature of the thick PBCO layer strongly improved its isolating properties, which is of extreme importance for good reproducability of junction fabrication. Special attention is being focused on the optimization of the contact of noble metal to YBCO as well its adhesion to the substrate. Best results are obtained using sputtered gold contacts, after a soft Ar ion sputter clean treatment of the damaged YBCO surface, followed by an anneal procedure.
\end{abstract}

\section{Introduction}

Josephson junctions made of high- $\mathrm{T}_{\mathrm{c}}$ materials are expected to be very attractive for building highly sensitive detectors for (sub-) millimeter wave radiation, because of their high energy gap values and the possibility to operate them at temperatures higher than $4.2 \mathrm{~K}$, up to $77 \mathrm{~K}$. Several studies have been reported showing very promising results of direct detection and mixing experiments in high $T_{c}$ Josephson junction devices, up to W-band frequencies, see, e.g., [1-6]. Most of the devices used in these studies, however, have properties that severely weaken their sensitivity as high frequency detectors, like low normal state resistances and strongly suppressed $I_{c} R_{n}$ products. For optimal performance, a large number of requirements must be met, which are presented in table 1.

In this contribution we report on the design and fabrication of a ramp-type $\mathrm{YBa}_{2} \mathrm{Cu}_{3} \mathrm{O}_{7}(\mathrm{YBCO}) /$ $\mathrm{PrBa}_{2} \mathrm{Cu}_{3} \mathrm{O}_{7}(\mathrm{PBCO}) / \mathrm{YBa}_{2} \mathrm{Cu}_{3} \mathrm{O}_{7}$ all high-Tc Josephson junction, integrated with a noble metal log-periodic antenna, suitable for high frequency applications. Optimization of junction preparation and the production of a metal antenna, with good electrical contact to YBCO and good adhesion to the substrate, will be discussed.

These investigations in the program of the Foundation for Fundamental Research on Matter (FOM) have been supported by the Netherlands Technology Foundation (STW).
Table 1.

Requirements for optimal device performance

Operating temperature $\mathrm{T}_{\text {op }} \sim 70 \mathrm{~K}$

Critical temperature $T_{c} \sim 85 \mathrm{~K}$

Critical current $I_{c}\left(T_{o p}\right) \gg$ noise current $I_{n}\left(T_{o p}\right)$

$$
\mathrm{I}_{\mathrm{n}, \text { thermal }}(77 \mathrm{~K}) \sim 3 \mu \mathrm{A}
$$

Normal state resistance $R_{n} \sim 10-100 \Omega$, for impedance matching to incoming radiation and output amplifiers

$I_{c} R_{n}$ products maximal, for optimal gain

RSJ like IV characteristics

Low noise

Substrates with low $\varepsilon_{\mathrm{T}}$ and $\tan \delta$, for low reflection and dielectric losses

Efficient, broadband coupling to (sub-) mm-wave radiation

\section{Design and Fabrication}

\section{$2.1 Y B C O / P B C O / Y B C O$ ramp junctions}

Ramp-type YBCO based Josephson junctions with an artificial PBCO barrier are used in this study. Details of the fabrication process have been published previously $[7,8]$. Shortly, a bilayer of YBCO/PBCO is deposited, forming the base electrode and the electrical isolation between the two superconducting electrodes. As substrate we use Yttria Stabilized $\mathrm{ZrO}_{2} \quad(100)$. The edge of the junction is created by low-angle $\left(20-25^{\circ}\right)$ Ar ion milling, to avoid possible polycrystallinity of the 
top electrode near the edge of the junction. After additional cleaning of the edge surface with a soft Ar ion beam, the thin PBCO barrier layer and the top YBCO electrode are deposited. The desired structure is subsequently defined by photolithographic patterning and Ar ion beam etching (Kaufman source, $3 \mathrm{~cm}$ beam width, $\mathrm{E}=500 \mathrm{eV}, \mathrm{I}=10 \mathrm{~mA}$ ), in a pulsed mode ( $8 \mathrm{sec}$. on, $10 \mathrm{sec}$. off), to avoid excessive heating of the substrate.

Scaling behavior of these junctions has been published [7,8]. It was found that the critical current density $J_{c}$ varies from $10^{1}-10^{3} \mathrm{~A} / \mathrm{cm}^{2}$ at $4.2 \mathrm{~K}$, and decreases exponentially for barrier thicknesses $\mathrm{L}$ larger than $6 \mathrm{~nm}$. The specific junction resistance $R_{n} A$ varies from $10^{-7}-10^{-5} \Omega \mathrm{cm}^{2}$, where $A$ is the junction area. For a given $J_{c}$ the corresponding $R_{n} A$ value is higher than for all other high- $T_{c}$ junctions published upto now. Using $L$ $15 \mathrm{~nm}$, an $R_{n} A$ value of about $10^{-6} \Omega \mathrm{cm}^{2}$ can be combined with $J_{c} \sim 150 \mathrm{~A} / \mathrm{cm}^{2}$, which agrees with the requirements.

Much work was done to optimize the junction fabrication, especially concerning the isolating $\mathrm{PBCO}$ layer. $\mathrm{PBCO}$, when deposited at the same temperature as $\mathrm{YBCO}$ (about $740^{\circ} \mathrm{C}$ ), grows heteroepitaxially on YBCO. Outgrowths that are initiated in the bottom YBCO electrode therefore, will continue to grow through the isolating PBCO layer, making it locally effectively thin. This causes a strong shunting of the junction region, reducing its $R_{n} A$ value and of course the reproducibility of the fabrication process. To solve this problem, the overlap area was minimized, to lower the chance of outgrowths being in there. Furthermore, the deposition temperature of the insulating $\mathrm{PBCO}$ layer was lowered to about $640^{\circ} \mathrm{C}$, at which the growth mechanism is different, though still epitaxial. Such a PBCO layer will stop the formation of outgrowths through the barrier and have a smoother surface by itself.

\section{2 coupling to millimeter waves}

To improve the coupling of high frequency radiation into the junction, it is placed between the rerminals of an antenna. In this study we use a self complementary planar log-periodic antenna, shown in figure 1. This type of antenna produces a nearly frequency independent, linearly polarized beam pattern, with maxima in the direction perpendicular to the plane of the antenna $[9,10]$. The covered frequency range is determined by the wavelengths resonating at the inner boundary of the smallest tooth, and at the outer boundary of the largest tooth. Mounted on a dielectric half sphere the antenna pattern is practically unidirectional, radiating predominantly through the dielectric. The impedance $Z$ of the antenna in such a configuration is real and given by $Z=377 /\left(2\left(1+\varepsilon_{\mathrm{r}}\right)\right)^{1 / 2}$ [11].

\section{3 integration of junction and antenna}

Recent studies of the surface resistance of YBCO show that at frequencies higher than about $100 \mathrm{GHz}$, the losses in YBCO are larger than those in a high conductivity normal metal [12]. For optimal sensitivity of the device it is desirable to have minimal losses in the antenna itself, so we chose to make it from metal. To avoid parasitic losses, the YBCO junction leads should have minimal area. Figure 2 shows the configuration near the terminals of the antenna.

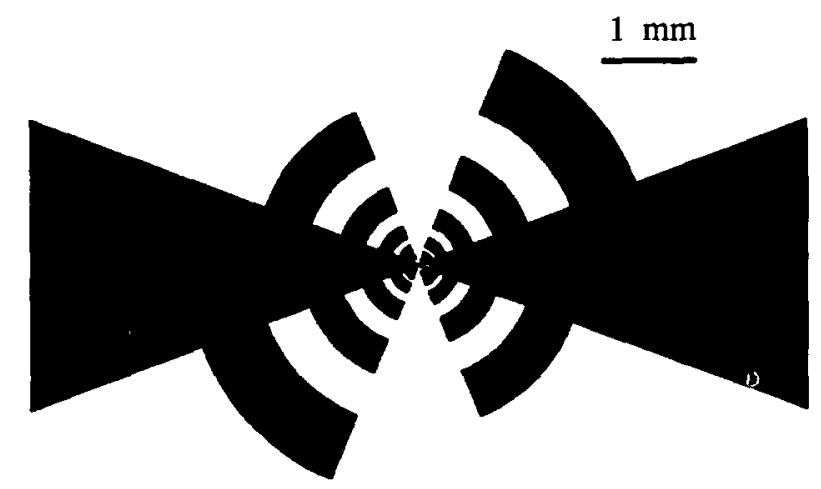

Figure 1. Log-periodic antenna.

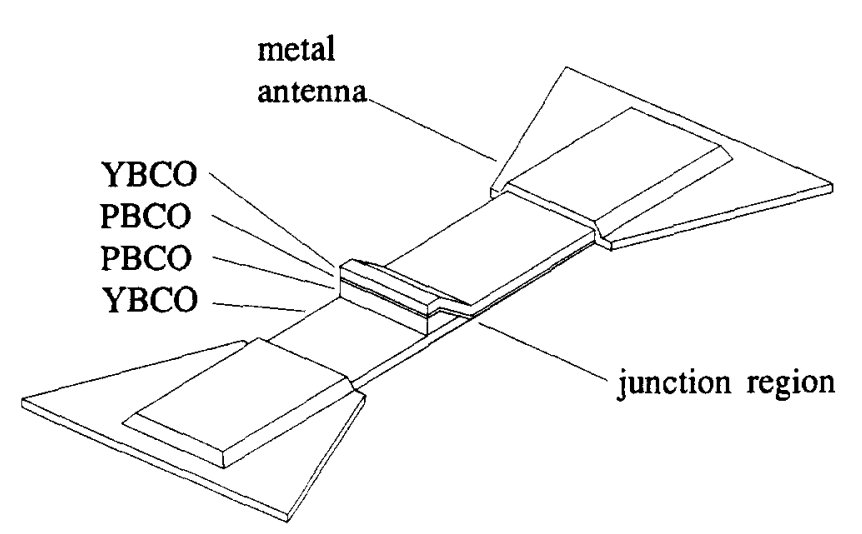

Figure 2. Terminal region of the antenna.

The process described in 2.1 is finished by structuring the junction leads, leaving a relatively large YBCO area near the junction region to avoid damage to the junction by exposure to various chemicals during the following processing steps. The device is then completed by depositing the metal film, as will be described in the next section, and structuring it into the antenna shape using lift off. The contact area between antenna terminals and junction leads is $30 \times 500 \mu \mathrm{m}^{2}$. In a final $\mathrm{Ar}$ ion beam etching step the shape of the junction is defined. 
Although for current-voltage (IV) measurements of the device a proper four terminal configuration is preferable, the presence of separate voltage leads will disturb the antenna pattern or increase the losses. Both current and voltage contacts will therefore be connected to the outer region of the antenna. This procedure gives rise to several fabrication problems. Firstly, the metal-YBCO contact resistance must be minimized and linearized, to avoid Joule heating, offset in IV measurements, and possible parasitic rectifying effects obscuring the properties of the junction itself. Secondly, since most of the metal is deposited directly on the substrate, good adhesion is required. To solve these problems, the optimimal conditions for metal deposition must be found.

\section{4 antenna fabrication}

The preparation of low ohmic metal contacts to YBCO thin films has also been studied by several other groups [13-15]. From these studies it is clear that the only materials easily available that do not reduce the YBCO material are $\mathrm{Au}$ and $\mathrm{Ag}$. The used deposition processes for both these materials are described in table 2. Procedures used for cleaning or annealing, to improve the electrical contacts, as well as the deposition of additional layers for improved adhesion, are also described.

The configuration described in 2.3 is not suited for a quantitative determination of contact resistivities [16], but in our case it does give a proper qualitative indication comparing several deposition techniques.

'As deposited' contacts, without any cleaning or annealing, are found to be very poor. Contact resistances $R_{c}$, measured at $77 \mathrm{~K}$, are about $10 \Omega$. Most likely, the surface layer of YBCO has metallike or even semiconducting properties due to multiple exposures to air and several chemicals during fabrication steps prior to the metal deposition [13-15]. The adhesion of the metals to the substrate is also poor. It never survived the simplest pulling test conceivable, using just a piece of adhesive tape.

Annealing of the $\mathrm{Ag}$ contact reduced $\mathrm{R}_{\mathrm{c}}$ by about one order of magnitude, probably because of strong $\mathrm{Ag}$ diffusion into the YBCO. The sticking to the substrate also improved. The surface of the $\mathrm{Ag}$ layer however, being smooth and shiny prior to the anneal, became rough and diffuse. This agrees with similar experiments reported in literature [15], where island formation in the $\mathrm{Ag}$ layer was found at temperatures above $370^{\circ} \mathrm{C}$. Annealing the $\mathrm{Au}$ contact also reduced the contact resistance slightly, although the sticking properties were still poor. The surface of the Au film remained mirror like.
Table 2. Procedures for $\mathrm{Ag} / \mathrm{Au}$ deposition $\mathrm{Ag} \quad \mathrm{Au}$

\begin{tabular}{|c|c|c|}
\hline method & $\begin{array}{l}\text { evaporation } \\
\text { pressure } \\
10^{-7} \text { to } \\
10^{-6} \text { mbar }\end{array}$ & $\begin{array}{l}\text { sputter } \\
\text { deposition } \\
\text { Ar pressure } \\
2.10^{-2} \text { mbar } \\
\text { power } 500 \mathrm{~W} \\
\text { target } \varnothing 20 \mathrm{~cm} \\
\text { self bias } 1350 \mathrm{~V}\end{array}$ \\
\hline thickness & $100 \mathrm{~nm}$ & $100 \mathrm{~nm}$ \\
\hline $\begin{array}{l}\text { annealing } \\
\text { conditions }\end{array}$ & $\begin{array}{c}1 \text { bar } \mathrm{O}_{2} \\
400^{\circ} \mathrm{C} \\
1 \text { hour }\end{array}$ & $\begin{array}{c}1 \text { bar } \mathrm{O}_{2} \\
500^{\circ} \mathrm{C} \\
1 \text { hour }\end{array}$ \\
\hline etch cleaning & not possible & $\begin{array}{l}\text { power } 150 \mathrm{~W} \\
\text { self bias } 350 \mathrm{~V}\end{array}$ \\
\hline $\begin{array}{r}\text { adhesion } \\
\text { layers }\end{array}$ & $\mathrm{Cr}, \sim 10 \mathrm{~nm}$ & $\mathrm{Ti}, 3-10 \mathrm{~nm}$ \\
\hline
\end{tabular}

To peal off the top layers of the damaged YBCO film and produce a new, fresh surface, soft $\mathrm{Ar}$ ion etching was used prior to metal deposition. In our case this was only possible in the Au deposition process. Using this cleaning procedure gave a bad, semiconducting contact. Only after additional annealing, $\quad R_{c}$ was lowered by two orders of magnitude compared to the 'as deposited' contacts. (Hahn et al. [13] suggested that more improvement could be obtained using a sputter gas containing $20 \%$ oxygen.) We also observed a significant improvement of the adhesion of Au to the substrate. Further studies will be directed, therefore, to the optimization of the cleaning parameters and the composition of the sputtergas.

Thin $\mathrm{Cr}$ and $\mathrm{Ti}$ films were used to improve the adhesion properties of $\mathrm{Ag}$ and $\mathrm{Au}$, respectively. They were deposited in situ, immediately followed by deposition of the $\mathrm{Ag} / \mathrm{Au}$ layers. Both in the $\mathrm{Au} / \mathrm{Ti}$ and in the $\mathrm{Ag} / \mathrm{Cr}$ case this gave a large improvement of the adhesion to the substrate. Since $\mathrm{Cr}$ and $\mathrm{Ti}$ chemically react with $\mathrm{YBCO}$, however, causing a strong degradation of the superconducting properties, direct contact of these materials to YBCO has to be avoided. Therefore, when an adhesion layer (AL) is involved, a bilayer technique is used. The first layer of $\mathrm{Ag} / \mathrm{Au}(\mathrm{M})$ is deposited directly on top of the $\mathrm{YBCO}$, and structured into the same shape as the junction leads. A thin $\mathrm{Cr} / \mathrm{Ti}$ layer is then deposited, directly followed by the second layer of $\mathrm{Au} / \mathrm{Ag}$. The result of this process is a $\mathrm{M} / \mathrm{AL} / \mathrm{M}$ structure on YBCO, giving a good electrical contact, and a $\mathrm{AL} / \mathrm{M}$ structure on the substrate, giving good adhesion there. 
This qualitative study suggests that good contacts may be obtained with evaporated $\mathrm{Ag}$, using a bilayer technique as described above with $\mathrm{Cr}$ as adhesion layer. Prior to deposition of the $\mathrm{Cr}$ and second $\mathrm{Ag}$ layer, the contact can be annealed (at temperatures below $370^{\circ} \mathrm{C}$ ), to improve $R_{c}$. Most promising results, however, have been obtained with sputtered Au, after soft $\mathrm{Ar}$ ion sputter cleaning of the YBCO surface, followed by an anneal treatment. This gives the lowest $R_{c}$ values and good adhesion to the substrate, even without using a bilayer design with $\mathrm{Ti}$ as sticking material. A quantitative study is being performed at this time, using a suitable structure for measuring contact resistivities. All cleaning, etching, deposition and annealing parameters of the two processes described will be optimized.

\section{Summary and Outlook}

Summarizing, we have described the design and fabrication of a device for highly sensitive detection of (sub-) millimeter waves. This device is a combination of an YBCO/PBCO/YBCO ramp type Josephson junction with a barrier thickness of 10-20 $\mathrm{nm}$ and a log-periodic antenna made of noble metal. These devices will meet the requirements for an efficient high-Tc high-frequency detector, concerning the values of critical current, normal state resistance and $I_{c} R_{n}$ products as well as efficient coupling to incoming radiation.

The deposition temperature of the isolating $\mathrm{PBCO}$ layer was lowered to prevent shunting of the junction by outgrowths in the base YBCO electrode.

A soft Ar ion sputter clean treatment prior to $\mathrm{Au}$ sputter deposition, to repair the damaged YBCO surface, was found to be the most promising procedure for obtaining low ohmic contacts of noble metal to the YBCO junction leads, as well as good adhesion of the metal to the substrate.

It is clear that much work is needed to meet all requirements for optimal properties of the described device. For example, the substrate should be changed from YSZ to a more suitable material like $\mathrm{LaAlO}_{3}$ or $\mathrm{NdGaO}_{3}$. This is likely to be the next step in the evolution process. Nevertheless, first junctionantenna combinations that were tested recently, show promising results. No degradation of junction properties due to the antenna patterning was observed. The devices showed good sensitivity to millimeter wave radiation: multiple Shapiro steps were observed at a frequency of $100 \mathrm{GHz}$ up to $75 \mathrm{~K}$, making operation possible in the submillimeter range. These results will be presented in a separate paper.

\section{References.}

[1] H.K. Olsson, W.R. Mcgrath, T. Claeson, S. Eriksson and L.-G. Johansson, J.Appl.Phys. 62 (1987) 4923.

[2] S. Kita, H. Tanabe and T. Kobayashi, IEEE Trans. MAG-25 (1989) 907.

[3] P.H. Wu, Y. Xu and C. Heiden, Appl.Phys.Lett. 57 (1990) 1265.

[4] Y. Fukumoto, H. Kajikawa, R. Ogawa and Y. Kawate, Jpn.J.Appl.Phys. 30 (1991), 3907.

[5] J.P. Hong, T.W. Kim, H.R. Fetterman, A.H. Cardona and L.C. Bourne, Appl.Phys.Lett. 59 (1991) 991.

[6] P.L. Richards and Q. Hu, Proc. IEEE 77 (1989) 1233.

[7] J. Gao, Yu.M. Boguslavskij, B.B.G. Klopman, D. Terpstra, G.J. Gerritsma, and H. Rogalla, Appl.Phys.Lett. 59 (1991) 2754.

[8] J. Gao, Yu.M. Boguslavskij, B.B.G. Klopman, D. Terpstra, R. Wijbrans, G.J. Gerritsma, and H. Rogalla, J.Appl.Phys. 72 (1992) 575.

[9] V.H. Rumsey, "Frequency Independent Antennas", Academic Press, New York, 1966.

[10] P.H. Siegel, IEEE MTT-S Digest (1986) 649.

[11] D.B. Rutledge, D.P. Neikirk and D.P. Kasillingam, "Integrated-circuit antennas", Infrared and $\mathrm{Mm}$ waves, vol. 10, 1-90, KJ. Button, ed., Academic Press, New York, 1983.

[12] A.A. Valenzuela, "Hochfrequenzeigenschaften supraleitender $\mathrm{YBa}_{2} \mathrm{Cu}_{3} \mathrm{O}_{7-\mathrm{x}}$ - dünnschichten", Ph.D. thesis, München, 1992, and references therein.

[13] R. Hahn, T. Schäffter, J. Klockau, G. Fotheringham, submitted to IEEE Trans. Applied Supercond. (ASC'92, Chicago, USA).

[14] H. Dreuth, "Eigenschaften des elektrischen Kontaktes zwischen $\mathrm{YBa}_{2} \mathrm{Cu}_{3} \mathrm{O}_{7-\mathrm{x}}$ und Normalmetallen", Universität Giessen, Diplomarbeit, 1992.

[15] Q.X. Jia, W.A. Anderson, J.P. Zheng, Y.Z. Zhu, S. Patel, H.S. Kwok, and D.T. Shaw, J.Appl.Phys. 68 (1990) 6336.

[16] W.M. Loh, S.E.Swirhun, T.A. Schreyer, R.M. Swanson, K.C. Saraswat, IEEE Trans. ED-34 (1987) 512. 